\title{
Máscaras de tecido na contenção de gotículas respiratórias - revisão sistemática
}

Homemade masks to contain respiratory droplets - systematic review Mascarillas caseras para contener gotas respiratórias - revisión sistemática

Monica Taminato ${ }^{1}$ (D) https://orcid.org/0000-0003-4075-2496)

Aline Mizusaki-Imoto² (iD https://orcid.org/0000-0001-8318-4658)

Humberto Saconato ${ }^{3}$ io https://orcid.org/0000-0002-0979-0286)

Eduardo Signorini Bicas Franco ${ }^{3}$ id https://orcid.org/0000-0003-2754-4369)

Maria Eduarda Puga ${ }^{4}$ (D https://orcid.org/0000-0001-8470-861X)

Márcio Luís Duarte ${ }^{4}$ io https://orcid.org/0000-0002-7874-9332)

Maria Stella Peccin ${ }^{4}$ io https://orcid.org/0000-0003-0329-4588)

Como citar:

Taminato M, Mizusaki-Imoto A, Saconato

$H$, Franco ES, Puga ME, Duarte ML, et al.

Máscaras de tecido na contenção de gotículas respiratórias - revisão sistemática. Acta Paul Enferm. 2020;33:AAPE20200103.

DOI

http://dx.doi.org/10.37689/actaape/2020AR0103

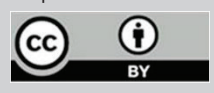

( ) infoccões: População; Dispositivos de proteção respiratória; Pandemia;

Máscaras

Keywords

COVID-19; Infection control; Population; Respiratory protective devices; Pandemics; Masks

Descriptores

COVID-19; Control de Infecciones; Población; Dispositivos de Protección Respiratoria;

Pandemias; Máscaras

Submetido 4 de Maio de 2020

Aceito

7 de Maio de 2020

Autor correspondente

Monica Taminato

E-mail: mtaminato@unifesp.br

\section{Resumo}

Objetivo: Identificar, avaliar sistematicamente e sumarizar as melhores evidências científicas disponíveis sobre a eficácia e a seguranç̧a das máscaras de tecido para a comunidade.

Métodos: Foram consultadas as bases de dados Cochrane, PUBMED, EMBASE, LILACS e a literatura cinzenta por meio do Opengrey. Também foi realizada busca nas referências bibliográficas dos estudos primários e secundários identificados. Não houve restrição de idioma, nem período de tempo. Foram incluídos todos os artigos que tenham como objetivo verificar a eficácia e segurança do uso de máscaras de tecido como proteção contra a transmissão viral, bem como estudos laboratoriais que avaliassem barreiras de contenção de partículas. Foram excluídos os estudos que envolvessem o uso de máscaras por profissionais de saúde. Dois avaliadores independentes selecionaram os estudos e as discrepâncias foram resolvidas por um terceiro avaliador.

Resultados: Após o processo de seleção, não foram localizados estudos clínicos randomizados envolvendo máscaras de tecidos para a população em geral. Incluímos sete estudos que avaliaram diferentes tecidos no bloqueio de gotículas de nível laboratorial e um estudo de revisão.

Conclusão: 0 uso de máscara de tecido possibilita uma barreira às gotículas quando comparada a nenhuma máscara. A máscara é um recurso adicional na prevenção e deve sempre ser associada à etiqueta respiratória, higienização das mãos, distanciamento social e isolamento dos casos.

\section{Abstract}

Objective: To identify, systematically review, and summarize the best scientific evidence available on the efficacy and safety of homemade cloth face masks for the community.

Methods: The search was conducted using the Cochrane, PUBMED, EMBASE, and LILACS databases, as well as grey literature, using Opengrey. A search was also conducted using references from primary and secondary studies that were found. No language or time restrictions were applied. All papers that objective was to check efficacy and safety of the use of cloth face masks as protection against viral transmission were included, as well as laboratory studies assessing barriers against particles. We excluded studies approaching the use of face masks by healthcare providers. Two independent reviewers selected the studies, and discrepancies were decided by a third reviewer.

Results: No randomized clinical trials involving cloth face masks for the general population were found. Seven studies assessing different types of cloth to prevent the penetration of droplets at a laboratory level and a review study were included.

Escola Paulista de Enfermagem, Universidade Federal de São Paulo, São Paulo, SP, Brasi

¿Escola Superior de Ciências da Saúde, Hospital das Forças Armadas, Brasília, DF, Brasil.

${ }^{3}$ Escola Paulista de Medicina, Universidade Federal de São Paulo, São Paulo, SP, Brasil.

“Universidade Federal de São Paulo, São Paulo, SP, Brasil.

Conflitos de interesse: Embora Taminato E seja Editora Associada da Acta Paulista de Enfermagem, ela não participou do processo de avaliação pelos pares do referido manuscrito. 
Conclusion: Using cloth face masks provides a barrier against droplets when compared with not using any face masks. The face mask is an additional preventive measure and must be used along with respiratory etiquette, hand hygiene, social distancing, and isolation of cases.

\section{Resumen}

Objetivo: Identificar, evaluar sistemáticamente y resumir las mejores evidencias científicas disponibles sobre la eficacia y la seguridad de las mascarillas caseras para la comunidad.

Métodos: Se consultaron las bases de datos Cochrane, PUBMED, EMBASE, LILACS y literatura gris por medio de Opengrey. También se realizó búsqueda en las referencias bibliográficas de los estudios primarios y secundarios identificados. No hubo restricción de idioma ni período de tiempo. Se incluyeron todos los artículos que tuvieran como objetivo verificar la eficacia y seguridad del uso de mascarillas caseras como protección contra la transmisión viral, así como estudios de laboratorio que evaluaran barreras de contención de partículas. Se excluyeron los estudios que abarcaran el uso de mascarillas por profesionales de la salud. Dos evaluadores independientes seleccionaron los estudios y las discrepancias fueron resueltas por un tercer evaluador.

Resultados: Luego del proceso de selección, no se localizaron estudios clínicos aleatorizados que incluyeran mascarillas caseras para la población en general. Incluimos siete estudios que evaluaron diferentes telas para el bloqueo de gotas de nivel de laboratorio y un estudio de revisión.

Conclusión: El uso de mascarillas caseras permite una barrera para las gotas al compararlo con ninguna mascarilla. La mascarilla es un recurso adicional en la prevención y siempre debe estar asociada a la etiqueta respiratoria, higienización de manos, distanciamiento social y aislamiento de casos.

\section{Introdução}

A Organização Mundial da Saúde (OMS) atualiza constantemente o número de casos de COVID-19 no mundo e o número de óbitos e os dados são alarmantes. Também informou que esforços de pesquisa e desenvolvimento estão avançando rapidamente em todo o planeta. ${ }^{(1)} \mathrm{O}$ chefe da OMS reitera a necessidade de buscar açóes e estratégias que possam ser alternativas para minimizar os efeitos desta pandemia, principalmente na população mais carente e na comunidade em geral. ${ }^{(2)}$ Frente a uma pandemia, há uma busca incansável por estratégias que possam orientar e minimizar o contágio na população e uso racional de equipamentos de proteção individual para os profissionais que estão na linha de frente, para tentar minimizar a disseminação na comunidade.

Ao considerarmos as doenças do Coronavírus (ex. síndrome respiratória aguda grave - SARS e a síndrome respiratória do Oriente Médio - MERS) e as experiências no controle e prevenção adotadas até o momento, as evidências apontam que o COVID-19 seja transmitido por meio de gotículas e modos de contato, sendo capaz ser transmitido por aerossóis relacionados aos procedimentos que produzem aerolização, tais como, coleta de amostra de swab, entubação orotraqueal, aspiração, entre outros. ${ }^{(3)}$

As indicaçóes do uso das máscaras para a população são baseadas nas experiências prévias no enfrentamento de síndromes respiratórias. No entanto, esta medida deve ser combinada com a higiene principalmente lavando as mãos, desinfecção das superfícies de muitos contatos, etiqueta de tosse e evitar tocar o rosto; todas as medidas combinadas são eficazes para impedir a transmissão humano-humano de 2019-nCov. ${ }^{(4)} \mathrm{O}$ uso de máscaras faciais para a população em geral pode atrasar uma pandemia por influenza e reduzir a taxa de ataque da infecção, podendo reduzir a transmissão o suficiente para conter a pandemia. ${ }^{(5)}$

Existem evidências indicando que o uso de máscaras pela população durante uma pandemia de uma doença respiratória pode minimizar a propagação da enfermidade e seu impacto econômico se usadas de maneira adequada e consistente, constituindo uma intervenção não farmacêutica eficaz no controle da propagação da doença. ${ }^{(6)} \mathrm{A}$ indicação de máscaras têxteis são sustentadas em pandemias e infecçóes emergentes, especialmente em contextos de baixa ou média renda.

Em virtude do risco aumentado de contágio numa pandemia como a do COVID-19, tendo em vista o alcance das gotículas e a emergência global da escassez de equipamentos de proteção individual, faz-se necessário identificar estratégias que possam promover a contenção das gotículas, minimizando riscos de infecções respiratórias na população em geral. O uso de máscaras de pano e reutilizáveis possuem importante aplicação para sintomáticos domiciliares, cuidadores e pessoas que moram em habitaçóes com muitos moradores, instituições de longa permanência e em espaços com aglomerações, como transportes públicos, por exemplo.

Desta forma, buscar identificar a efetividade e a segurança de máscaras de tecido para esta população é de extrema importância. 
O objetivo desta revisão sistemática é identificar a efetividade das evidências científicas acerca do uso de máscaras de tecido para a comunidade como medida não farmacológica para controle de infecção por COVD-19 para a população geral.

\section{Métodos}

\section{Desenho e local do estudo}

Esta foi uma revisão sistemática, de escopo, desenvolvida por pesquisadores do Centro Cochrane do Brasil, pelo Grupo de Pesquisa Epidemiológica, Revisão Sistemática e Políticas de Saúde, CNPQ da Escola Paulista de Enfermagem, pelo Grupo de Pesquisa do CNPQ de Prática Baseada em Evidências, Programa de Pós-graduação em Saúde Baseada em Evidências da UNIFESP e Programa de Mestrado Profissional e Acadêmico da Escola Superior de Ciências da Saúde (ESCS), Hospital das Forças Armadas. A revisão foi registrada na base Open Science Framework em 04/04/2020.

\section{Critérios de Elegibilidade}

\section{Tipos de Participantes}

Foram incluídos estudos que envolvessem adultos, crianças e idosos da comunidade em geral.

\section{Tipos de Intervençáo}

Foi considerado como critério de elegibilidade estudos que envolvessem máscaras de diferentes tipos de tecidos (que serão adaptadas à face e cobrindo a região da boca e nariz).

\section{Tipos de Estudos}

Considerando o número limitado de estudos sobre máscaras de tecidos para comunidade em geral, o objetivo desta revisão rápida, será mapear o conhecimento sobre o assunto e identificar os desenhos de estudos por nível de evidências.

\section{Tipos de Desfechos}

Os desfechos selecionados como primários foram: contenção de gotículas, incidência de infecçôes respiratórias e eventos adversos. Como desfechos secundários: internação hospitalar, curva de crescimento de novos casos e mortalidade.

\section{Busca por estudos}

As buscas foram construídas utilizando os Descritores em Ciências da Saúde e traduzidas para cada uma das bases de dados selecionadas: Cochrane Library (Wiley); Embase (Elsevier); Portal BVS; Medical Literature Analysis and Retrieval System Online (MEDLINE, PubMed); CINAHL; Web of Science; Scopus; Opengrey (https://opengrey.eu) Literatura cinzenta. Foi utilizada a busca manual nas referências dos estudos primários e secundários identificados na busca eletrônica. As estratégias de busca elaboradas e utilizadas para cada base eletrônica de dado estão apresentadas no anexo 1 e foram realizadas em 20 de abril de 2020 e náo houve restrição a idiomas ou formas de publicação.

\section{Seleção dos estudos}

O processo de seleção dos estudos foi realizado por dois revisores independentes e qualquer divergência foi resolvida por um terceiro avaliador. A seleção dos estudos foi realizada em duas etapas. $\mathrm{Na}$ primeira etapa foram avaliados os títulos e resumos das referências identificadas por meio da estratégia de busca e os estudos potencialmente elegíveis foram pré-selecionados. $\mathrm{Na}$ segunda etapa, foi realizada a avaliação do texto na íntegra dos estudos pré-selecionados para confirmação da elegibilidade. O processo de seleção foi realizado por meio da plataforma Rayyan (https://rayyan.qcri.org) (Anexo 2).

\section{Resultados}

As estratégias de busca recuperaram 1237 referências. Durante o processo de seleção, foram eliminadas 383 referências duplicadas (referências idênticas) e 820 referências que não estavam de acordo com os critérios de inclusão após a leitura do título e do resumo (primeira etapa). A leitura do texto completo das 34 referências selecionadas confirmou a elegibilidade (segunda etapa). O fluxograma do processo de seleção está apresentado na figura 1. Após o processo de seleção, 07 estudos foram incluídos. 


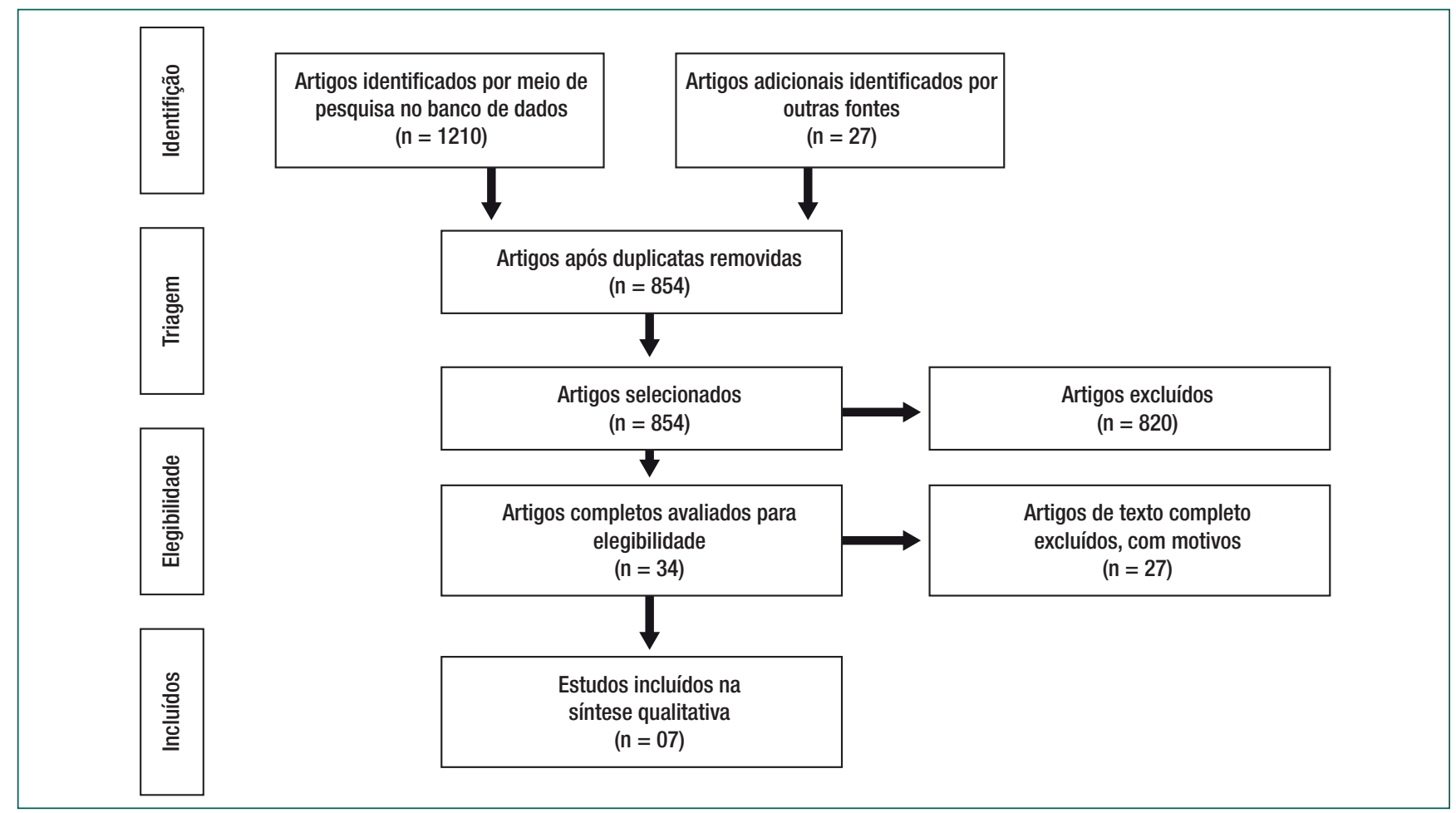

Figura 1. Fluxo de seleção dos artigos

A leitura do texto completo das 34 referências resultou na exclusão de 27 estudos por não se adequarem aos critérios de inclusão desta pesquisa. Restaram sete estudos cujos resultados serão apresentados em formato descritivo, pois impossibilitam qualquer outro tipo de análise.

\section{Característica dos estudos incluídos}

Os sete estudos incluídos nesta revisão foram publicados nos anos de 1983, 2008, 2010 (2), 2013, 2016 e 2020. Os países de origem foram um do Reino Unido, quatro dos Estados Unidos e dois da Holanda. Quanto ao tipo de estudos tivemos cinco estudos laboratoriais, um experimental e um estudo de revisão quadro 1 .

Estudos laboratoriais foram incluídos nesta revisão rápida para verificar a eficácia da incorporação deste tipo de material para contenção de gotículas na população geral. Todos os estudos avaliaram a eficácia do tecido em relação às máscaras N95 e cirúrgicas. Os resultados apontam superioridade para as máscaras indicadas para uso de profissionais de saúde e verificam que dependendo do tipo de tecido, mesmo com eficácia inferior, são capazes de conter mais de $90 \%$ das gotículas.
O primeiro estudo (1983) testou diferentes tipos de materiais em um manequim conectado a um simulador de respiração para determinar a fração de aerossol de 2 micrômetros de diâmetro e a eficácia dos materiais. Foram testados: algodão (tecido de camiseta), tecido usado em lenço, tecido atoalhado e máscara cirúrgica. Em uma frequência respiratória de 37 litros por minuto, a média de escape variou de 0 a 63\% e a média de penetração de partículas variou de $0.6 \%$ a $39 \%$. O uso de nylon para prender o material do lenço ou a máscara facial descartável na face mostrou-se muito eficaz na prevenção de dispersão. Pode-se esperar que essa combinação reduza a dispersão em torno do lenço para cerca de $10 \%$ ou menos na prática e em torno da máscara para menos de um por cento. ${ }^{(7)}$

O estudo de Van der Sande $(2008)^{8}$, realizou um experimento comparando três tipos de máscaras: uma Máscara FFP2, uma máscara cirúrgica e uma máscara caseira. Incluíram 28 adultos voluntários e 11 crianças entre 5-11 anos voluntárias. Neste protocolo padrão, o voluntário foi solicitado a executar cinco tarefas sucessivas em uma sequência 1,5 minuto de duração cada: sem atividade - sente-se, acene cabeça ("sim”), sacuda a cabeça ("não"), leia 
Quadro 1. Descrição dos estudos incluídos

\begin{tabular}{|c|c|c|c|c|}
\hline Artigo & $\begin{array}{l}\text { Desenho } \\
\text { estudo }\end{array}$ & Métodos & Resultados & Conclusões \\
\hline $\begin{array}{l}\text { 1. Cooper DW, Hinds WC, Price } \\
\text { JM, Weker R, Yee HS. Common } \\
\text { materials for emergency respiratory } \\
\text { protection: Leakage tests with } \\
\text { a manikin. Am Ind Hyg Assoc J. } \\
\text { 1983;44(10):720-6. (") }\end{array}$ & Laboratorial. & $\begin{array}{l}\text { Teste feito em um manequim conectado } \\
\text { a um simulador de respiração para } \\
\text { determinar a fração de aerossol de } 2 \\
\text { micrômetros de diâmetro e a eficácia } \\
\text { de diferentes materiais. Foi utilizado } \\
\text { tecido de camisa de algodão/poliester, } \\
\text { lenço de algodão, tecido de toalha, uma } \\
\text { máscara cirúrgica e uma máscara facial } \\
\text { descartável. }\end{array}$ & $\begin{array}{l}\text { Em uma frequência respiratória de } \\
37 \text { litros por minuto, a média de } \\
\text { escape variou de } 0 \text { a } 63 \% \text { e a média } \\
\text { de penetração de partículas variou } \\
\text { de } 0.6 \% \text { a } 39 \% \text {. } 0 \text { uso de nylon para } \\
\text { prender o material do lenço ou a } \\
\text { máscara facial descartável na face } \\
\text { mostrou-se muito eficaz na prevenção } \\
\text { de dispersão. }\end{array}$ & $\begin{array}{l}0 \text { uso de material de meias de nylon ("meia- } \\
\text { calça") para prender o material do lenço } \\
\text { ou a máscara facial descartável na face } \\
\text { foi considerado muito eficaz na prevenção } \\
\text { de vazamentos. Pode-se esperar que essa } \\
\text { combinação reduza o vazamento em torno do } \\
\text { lenço para cerca de } 10 \% \text { ou menos na prática } \\
\text { e em torno da máscara para menos de um } \\
\text { por cento. }\end{array}$ \\
\hline $\begin{array}{l}\text { 2. van der Sande M, Teunis } P \text {, } \\
\text { Sabel R. Professional and home- } \\
\text { made face masks reduce exposure } \\
\text { to respiratory infections among } \\
\text { the general population. PLoS One. } \\
2008 ; 3(7): \text { e2618. }^{(8)}\end{array}$ & Laboratorial. & $\begin{array}{l}\text { Testou três tipos de máscaras diferentes, } \\
\text { sendo duas profissionais e uma máscara } \\
\text { caseira feita com pano de chá. Os } \\
\text { indivíduos faziam tarefas específicas e a } \\
\text { concentração de partículas era medida } \\
\text { em ambos os lados da máscara. }\end{array}$ & $\begin{array}{l}0 \text { estudo aponta para a superioridade } \\
\text { da FFP2 seguida das máscaras } \\
\text { cirúrgicas e menor eficácia para as } \\
\text { máscaras caseiras, mostrando que } \\
\text { um importante fator para diminuição } \\
\text { da eficácia é o tempo de uso e sua } \\
\text { relação com a umidade. }\end{array}$ & $\begin{array}{l}\text { Todas as máscaras ofereciam proteção contra } \\
\text { a transmissão, reduzindo exposição durante } \\
\text { todos os tipos de atividades, para crianças e } \\
\text { adultos. }\end{array}$ \\
\hline $\begin{array}{l}\text { 3. Rengasamy S, Eimer B, Shaffer } \\
\text { RE. Simple respiratory protection- } \\
\text { evaluation of the filtration } \\
\text { performance of cloth masks and } \\
\text { common fabric materials against } \\
\text { 20-1000 nm size particles. } \\
\text { Annals of occupational hygiene. } \\
\text { 2010;54(7):789-98. }{ }^{(9)}\end{array}$ & Laboratorial. & $\begin{array}{l}\text { Avaliou os diferentes tipos de tecido: } \\
\text { moletom, camiseta, toalha e lenço } \\
\text { comparados com a máscara N95 na } \\
\text { capacidade de filtragem de partículas em } \\
\text { diferentes velocidades. }\end{array}$ & $\begin{array}{l}\text { Toalhas e echarpes: } 60-66 \% \text { e } \\
\text { 73-89\%. Moletom: } 30-61 \% \text { para } \\
\text { partículas de 20nm, aumentando para } \\
80-93 \% \text { para partículas de } 1000 \mathrm{~nm} \text {. } \\
\text { Camiseta: } 56-79 \% \text { para partículas } \\
\text { de } 20 \mathrm{~nm} \text { e } 89-97 \% \text { para partículas } \\
\text { de } 1000 \mathrm{~nm} \text {. Toalhas e echarpes: } 9 \text { a } \\
74 \% \text { para partículas de } 20 \mathrm{~nm} \text {. }\end{array}$ & $\begin{array}{l}\text { Os resultados mostraram que as máscaras } \\
\text { de tecido de moletom e toalha mostraram } \\
\text { menores níveis de passagem quando } \\
\text { comparadas aos outros tipos de tecido. Todas } \\
\text { as análises demonstraram inferioridade da } \\
\text { capacidade de filtragem das máscaras de } \\
\text { tecido em relação a máscara N95. }\end{array}$ \\
\hline $\begin{array}{l}\text { 4. Brienen NC, Timen A, Wallinga J, } \\
\text { van Steenbergen JE, Teunis PF. The } \\
\text { effect of mask use on the spread of } \\
\text { influenza during a pandemic. Risk } \\
\text { Anal. 2010;30(8):1210-8.(5) }\end{array}$ & Revisão. & $\begin{array}{l}\text { Reflexão acerca da necessidade de } \\
\text { implementação de medidas de contenção } \\
\text { farmacêuticas (vacinas e antivirais) e não } \\
\text { farmacêuticas para a preparação de uma } \\
\text { pandemia iminente. }\end{array}$ & $\begin{array}{l}\text { Estudos retrospectivos de caso- } \\
\text { controle mostraram que o uso } \\
\text { de máscaras pela população em } \\
\text { geral pode ter oferecido proteção } \\
\text { significativa contra a SARS. }\end{array}$ & $\begin{array}{l}0 \text { uso de máscaras faciais para a população } \\
\text { geral pode atrasar uma pandemia por influenza } \\
\text { e reduzir a taxa de ataque da infecção, } \\
\text { podendo reduzir a transmissão o suficiente } \\
\text { para conter a pandemia. } 0 \text { efeito no tamannho } \\
\text { final da epidemia depende das características } \\
\text { da transmissão do vírus, da eficiência da } \\
\text { máscara e da cobertura do uso da máscara } \\
\text { pela população. }\end{array}$ \\
\hline $\begin{array}{l}\text { 5. Davies A, Thompson K, Giri K, } \\
\text { Kafatos G, Walker J, Bennet A.T } \\
\text { Testing the efficacy of homemade } \\
\text { masks: would they protect in an } \\
\text { influenza pandemic? Disaster } \\
\text { Med Public Health Prep. 2013; } \\
\text { 7(4):413-8. }{ }^{\text {(10) }}\end{array}$ & Laboratorial. & $\begin{array}{l}21 \text { voluntários saudáveis fizeram suas } \\
\text { máscaras em casa com tecido } 100 \% \\
\text { algodão. Com várias técnicas de } \\
\text { amostragem de ar, os microrganismos } \\
\text { da tosse desses voluntários foram } \\
\text { isolados e o uso da máscara caseira foi } \\
\text { comparada com a máscara cirúrgica } \\
\text { ou sem máscara. Foram testadas a } \\
\text { capacidade de filtração e de contenção } \\
\text { de microrganismos. }\end{array}$ & $\begin{array}{l}0 \text { principal resultado encontrado } \\
\text { neste estudo foi a capacidade de } \\
\text { contenção dos microrganismos } \\
\text { nos diferentes tipos de tecidos. } 0 \\
\text { estudo demonstra que o uso das } \\
\text { máscaras caseiras é adequado para } \\
\text { a população e que não devem ser } \\
\text { utilizadas pelos os profissionais de } \\
\text { saúde. }\end{array}$ & $\begin{array}{l}\text { Houve contenção dos microrganismos nos } \\
\text { diferentes tecidos, sendo que a máscara } \\
\text { cirúrgica foi } 3 \text { vezes melhor. } 0 \text { uso de } \\
\text { máscaras caseiras é adequado para a } \\
\text { população e que não devem ser utilizadas } \\
\text { pelos os profissionais de saúde. }\end{array}$ \\
\hline $\begin{array}{l}\text { 6. Shakya KM, Noyes A, Kallin R, } \\
\text { Peltier RE. Evaluating the efficacy } \\
\text { of cloth facemasks in reducing } \\
\text { particulate matter exposure. } \\
\text { J Expo Sci Environ Epidemiol. } \\
\text { 2017;27(3):352-7.(11) }\end{array}$ & Laboratorial. & $\begin{array}{l}\text { Estudo comparativo sobre a retenção } \\
\text { de partículas por diferentes tipos de } \\
\text { máscaras. Avaliou três tipos de máscaras } \\
\text { de pano e um tipo de máscara cirúrgica. } \\
\text { Foram utilizados cinco tamanhos de } \\
\text { esfera de aerossol monodispersa e } \\
\text { exaustão de diesel diluído. }\end{array}$ & $\begin{array}{l}\text { Entre os três tipos de máscaras } \\
\text { de pano, uma máscara de pano } \\
\text { com válvula de escape teve um } \\
\text { desempenho melhor. Uma máscara } \\
\text { N95 foi utilizada como controle para } \\
\text { comparar os resultados com as } \\
\text { máscaras de pano e os resultados } \\
\text { sugerem que as máscaras de pano } \\
\text { são apenas marginalmente benéficas } \\
\text { na proteção dos indivíduos contra } \\
\text { partículas menores que } 2,5 \text { ㅆm. }\end{array}$ & $\begin{array}{l}\text { A máscara N95 é superior na retenção } \\
\text { de partículas, mas as demais máscaras } \\
\text { também apresentam sua eficieincia, mas } \\
\text { em percentuais marginais. Comparadas às } \\
\text { máscaras de pano, as máscaras cirúrgicas } \\
\text { descartáveis são mais eficazes na redução da } \\
\text { exposição a partículas. }\end{array}$ \\
\hline $\begin{array}{l}\text { 7. Konda A, Prakash A, Moss } \\
\text { G, Schmoldt, Gregory D. Grant, } \\
\text { Guha M. } \\
\text { Aerosol filtration efficiency of } \\
\text { common fabrics used in respiratory } \\
\text { cloth masks. ACS Nano. } 2020 \text { Apr } \\
\text { 24:acsnano.0c03252:(12) }\end{array}$ & $\begin{array}{l}\text { Estudo } \\
\text { transversal. }\end{array}$ & $\begin{array}{l}\text { Testa } 15 \text { tipos diferentes de tecido de } \\
\text { máscaras. }\end{array}$ & $\begin{array}{l}\text { Foram medidas três variações: } \\
\text { combinando uma camada de algodão } \\
600 \text { TPI com duas camadas de seda, } \\
\text { duas camadas de chi囚on e uma } \\
\text { camada de flanela. Os resultados } \\
\text { também são comparados com o } \\
\text { desempenho de uma máscara N95 } \\
\text { padrão. }\end{array}$ & $\begin{array}{l}\text { Duas camadas de algodão } 600 \text { TPI é } \\
\text { claramente superior com mais de } 65 \% \text { de } \\
\text { eficiência em partículas menores que } 300 \mathrm{~nm} \\
\text { e mais de } 90 \% \text { de eficiência em partículas } \\
\text { maiores que } 300 \mathrm{~nm} \text {. } \\
\text { A colcha forneceu excelente filtragem em } \\
\text { vários tamanhos de partículas (mais de } 80 \% \\
\text { para partículas menores que } 300 \mathrm{~nm} \text { e mais } \\
\text { de } 90 \% \text { para partículas maiores que } 300 \mathrm{~nm} \text { ). } \\
0 \text { desempenho de um composto de seda com } \\
\text { quatro camadas oferece mais de } 80 \% \text { de } \\
\text { eficiência de filtragem em toda a faixa de } 10 \\
\mathrm{~nm} \text { a } 6 \text { um. }\end{array}$ \\
\hline
\end{tabular}


em voz alta um texto padrão, caminhada estacionária. Ao longo deste exercício, a concentração das partículas foi medida em ambos os lados da máscara através de um receptor fixo na face e no lado externo da máscara. O estudo aponta para a superioridade da FFP2 seguida das máscaras cirúrgicas e com menor eficácia para as máscaras caseiras, mostrando que um importante fator para diminuição da eficácia é o tempo de uso e a sua relação com a umidade. Todas as máscaras ofereciam proteção contra a transmissão, reduzindo a exposição durante todos os tipos de atividades, para crianças e adultos. Dentro de cada categoria de máscaras, o grau de proteção variou por categoria de idade e, em menor grau, por atividade. Não observaram diferenças entre homens e mulheres. ${ }^{(8)}$

Em seguida, o outro estudo encontrado (2010), o enfoque foi avaliar os diferentes tipos de tecido: moletom, camiseta, toalha e lenço comparados com a máscara N95. Os resultados mostraram que as máscaras de tecido de moletom e toalha mostraram menores níveis de passagem quando comparadas aos outros tipos de tecido. Todas as análises demonstraram inferioridade da capacidade de filtragem das máscaras de tecido em relação a máscara N95. Toalhas e echarpes: $60-66 \%$ e $73-89 \%$. Moletom: 30-61\% para partículas de $20 \mathrm{~nm}$, aumentando para 80-93\% para partículas de 1000 nm. Camiseta: $56-79 \%$ para partículas de $20 \mathrm{~nm}$ e 89-97\% para partículas de $1000 \mathrm{~nm}$. Toalhas e echarpes: 9-74\% para partículas de $20 \mathrm{~nm} .{ }^{9)}$

O quarto estudo encontrado (2010), faz uma reflexão acerca da necessidade de implementação de medidas de contenção farmacêuticas (vacinas e antivirais) e não farmacêuticas para a preparação de uma pandemia iminente. Uma vez que suprimentos farmacêuticos adequados não estejam disponíveis e haja também carência de evidências científicas, a OMS recomenda, como intervenção pública na saúde, intervenções não farmacêuticas como medidas de controle adicional. Estas medidas têm como objetivos limitar a disseminação internacional do vírus, reduzir a propagação dentro das populações, reduzir o risco individual de infecção (através de medidas de proteção e higiene pessoal) e conscientizar o público sobre os riscos. As másca- ras tradicionalmente são usadas há séculos. Estudos retrospectivos de caso-controle mostraram que o uso de máscaras pela população em geral pode ter oferecido proteção significativa contra a SARS 5 . O uso de máscaras faciais para a população geral pode atrasar uma pandemia por influenza e reduzir a taxa de ataque da infecção, podendo reduzir a transmissão o suficiente para conter a pandemia. $\mathrm{O}$ efeito no tamanho final da epidemia depende das características da transmissão do vírus, da eficiência da máscara e da cobertura do uso da máscara pela população. Os achados de Brienen são baseados em dados da literatura publicada e de modelos matemáticos. ${ }^{(5)}$

O quinto estudo (2013), teve como objetivo avaliar as máscaras caseiras como alternativas às máscaras comerciais. Vinte e um voluntários saudáveis, 12 homens e 9 mulheres, com idade entre $20 \mathrm{e}$ 44 anos, fizeram suas máscaras em casa, com tecido de camisetas de 100\% algodão, usando máquinas de costura. Com várias técnicas de amostragem de ar, os microrganismos da tosse desses voluntários foram isolados usando máscara caseira de tecido de algodão, máscara cirúrgica ou sem máscara ${ }^{10}$. Foram avaliados diferentes tipos de tecidos utilizados para confecção de máscaras caseiras comparadas às máscaras cirúrgicas. Foram testadas a capacidade das máscaras de filtração e a contenção de microrganismos, utilizando testes microbiológicos. $\mathrm{O}$ principal resultado encontrado neste estudo foi a capacidade de contenção dos microrganismos nos diferentes tipos de tecidos. No entanto a máscara cirúrgica apresentou uma efetividade 3 vezes superior. $\mathrm{O}$ estudo demonstra que o uso das máscaras caseiras são adequadas para a população e que não devem ser utilizadas pelos os profissionais de saúde. ${ }^{(10)}$

O estudo de Shakya KM (2016) avaliou três tipos de máscaras de pano e um tipo de máscara cirúrgica. Foram utilizados cinco tamanhos de esfera de aerossol monodispersa e exaustão para os testes. Entre os três tipos de máscaras de pano, uma máscara de pano com válvula de escape teve um desempenho melhor. Uma máscara N95 foi usado como controle para comparar os resultados. A máscara N95 foi superior às três máscaras de tecido para todas as partículas. Em um fluxo baixo, a máscara de tecido a cirúrgica foram similares à N95. A eficiên- 
cia da máscara cirúrgica foi levemente melhor que a máscara de tecido 1. Comparadas às máscaras de pano, as máscaras cirúrgicas descartáveis são mais eficazes na redução da exposição a partículas. ${ }^{(11)}$

No estudo de Konda 2020 foram medidas três variaçôes: combinando uma camada de algodáo 600 TPI com duas camadas de seda, duas camadas de chiffon e uma camada de flanela. Os resultados também foram comparados com o desempenho de uma máscara N95 definido como padrão. Todas as três combinações tiveram bom desempenho, excedendo $80 \%$ de eficiência nas partículas menores que $300 \mathrm{~nm}$ e mais de $90 \%$ nas partículas maiores que $300 \mathrm{~nm}$. As combinaçóes de tecido são ligeiramente inferiores à máscara N95. São preferidos tecidos de baixa porosidade, como os encontrados em lençóis de algodão com alta contagem de linhas. Materiais como seda natural, chiffon e flanela podem fornecer um bom filtro eletrostático de partículas. Combinar camadas para formar máscaras híbridas, alavancar o filtro mecânico e eletrostático pode ser uma abordagem eficaz. A eficiência da filtragem era maior que $80 \%$ para partículas menores que $300 \mathrm{~nm}$ e maiores que $90 \%$ para partículas maiores que $300 \mathrm{~nm}$. Em resumo, o estudo aponta que o uso de máscaras de pano combinado com diferentes tipos de tecidos pode fornecer proteção significativa contra a transmissão de partículas na faixa de tamanho de aerossol. ${ }^{(12)}$

\section{Discussão}

A principal contribuição desta revisão rápida é subsidiar as indicaçóes do Ministério da Saúde para o uso de máscaras e sintetizar as melhores evidências disponíveis para a indicação desta intervenção não farmacológica associada às medidas de etiqueta de tosse e higienização das mãos baseadas no princípio de proteção individual.

Tais indicações são oportunas como medida social de baixo custo para a população geral, especialmente para o momento em que questóes sobre a transmissibilidade de assintomáticos e oligossintomáticos não estão totalmente definidas bem como uma intervenção para os países pouco desenvolvidos e em desenvolvimento que apresentam sérios problemas sociais, principalmente em relação à pre- cariedade das moradias e a impossibilidade de distanciamento social. ${ }^{(13,14)}$

A indicação do uso de máscaras para a contenção de gotículas foi indicada em outras emergências globais, como em 2009 na pandemia de H1N1, como uma medida de estratégia não farmacológica de prevenção e mitigação de infecções transmitidas por gotículas, tornando-se uma consideração importante no desenvolvimento de políticas em torno do uso de máscaras não médicas pela população. $(15,16)$

O uso de máscaras de pano e reutilizáveis possuem importante aplicação para sintomáticos domiciliares, cuidadores e pessoas que moram em habitaçóes com muitos moradores, instituiçôes de longa permanência e em espaços com aglomeraçóes, como transportes públicos, por exemplo.

Dentre os ensaios clínicos encontrados que avaliaram o uso de máscaras para a população e familiares para evitar a transmissão por gotículas na pandemia do $\mathrm{H} 1 \mathrm{~N} 1$, todos os que utilizaram máscaras cirúrgicas foram excluídos, pois não preencheram ao critério de inclusão sobre o tipo de material. Nosso enfoque foi no uso de máscaras de tecido para responder a uma emergência global acerca da falta de equipamentos de proteção individual para profissionais de saúde.

Dentre os estudos avaliados e excluídos por utilizarem máscaras cirúrgicas na população, todos demonstram impactos na redução da transmissibilidade entre familiares e comunidade quando associadas às medidas de etiqueta de tosse e higienizaçáo das mãos.

O uso de máscaras não apenas protege indivíduos saudáveis, mas também reduz a infectividade de sintomáticos e assintomáticos, reduzindo assim o número e a eficácia das fontes de transmissão na população. Além do mais, é esperado que o uso das máscaras seja influenciado no comportamento da população, aumentando a conscientização sobre a infecção, que representa a importância de comportamentos preventivos adicionais, como lavar as mãos com maior frequência, evitar contato físico, evitar aglomeraçóes e locais públicos lotados. Soma-se a isso o fato da máscara impedir que os usuários toquem a boca ou o nariz com as mãos ou outros objetos potencialmen- 
te contaminados pelo vírus. No entanto, por outro lado, o uso das máscaras faciais pode gerar uma falsa sensaçáo de segurança e levar ao uso reduzido de outras medidas, como a higiene pessoal.

Um relato de caso sobre o impacto do uso de máscaras em um transporte público durante a pandemia de COVID-19 na China, descreveu o potencial de transmissão para 5 pessoas na ausência do uso de máscaras. ${ }^{(17)}$

O governo chinês instituiu várias medidas para controle da pandemia como o bloqueio de cidades, desligamento do sistema de transporte, fechamento de escolas e recomendaçóes de higiene das mãos e máscaras para todos. ${ }^{(18-20)}$ Assim que a população em geral começou a perceber a gravidade do surto, o consumo de máscaras aumentou em poucos dias, em parte devido à falta de informaçóes sobre o novo vírus e ao pânico, gerando um grande problema de desabastecimento para os profissionais de saúde.

Diante de tais circunstâncias, diversos governos emitiram recomendaçôes para uso de máscaras faciais caseiras. Identificamos como implicação para a pesquisa a necessidade de estudos clínicos randomizados para avaliar a efetividade e a aderência da utilização de máscaras caseiras na comunidade. ${ }^{(21)}$

Como implicação para a prática, consideramos que os estudos apresentam que alguns tecidos utilizados em mais de uma camada podem atuar como uma barreira parcial a gotículas e, em situaçóes de pandemia como a que o mundo vive hoje, deve ser um recurso associado as demais medidas de higienização das mãos e distanciamento social.

Assim, qualquer máscara, não importa quão eficiente na filtragem ou quão boa é a vedação, terá um mínimo efeito se não for utilizada associada as outras medidas, como isolamento de casos, distanciamento social, boas práticas de etiqueta respiratória e higienização regular das mãos.

\section{Conclusão}

Não identificamos estudos clínicos que avaliassem a efetividade de máscaras feitas em casa para redução da emissão de partículas e prevenção de infecção respiratória. Os estudos, na sua maioria laboratoriais, ava- liam as diferenças dos tecidos em relação a contenção de gotículas, comparando-as com as máscaras profissionais, normalmente cirúrgicas. Há diferença em termos de proteção proporcionada por uma máscara cirúrgica e uma máscara de tecido caseira. Porém, observou-se que existe diferença em relação a contenção de gotículas quando se utiliza algum tipo de máscara ou nenhuma máscara. Os estudos demonstram que a máscara é um recurso a mais na prevenção, que deve sempre vir acompanhada do distanciamento social, medidas de higienização das mãos e etiqueta respiratória. Diante do risco de transmissão por assintomáticos, dos desafios em saúde pública relacionados as questôes sociais e da necessidade de retorno gradativo e programado das atividades, sugerimos que medidas destinadas a melhorar a higienização das mãos, distanciamento social e o uso de máscaras pela população sejam consideradas como estratégia não farmacológica efetiva na prevenção para o COVID-19.

\section{Referências}

1. Brasil. Ministério da Saúde. Nota Informativa №3/2020-CGGAP/DESF/ SAPS/MS A Lei n 13.969. Brasília (DF): Ministério da Saúde; 2020 Feb $6.3 \mathrm{p}$.

2. Maclntyre CR, Seale H, Dung TC, Hien NT, Nga PT, Chughtai AA, et al. A cluster randomised trial of cloth masks compared with medical masks in healthcare workers. BMJ Open. 2015;5(4):e006577.

3. To KK, Tsang OT, Chik-Yan Yip C, Chan KH, Wu TC, Chan JM, et al. Consistent detection of 2019 novel coronavirus in saliva. Clin Infect Dis. 2020 Feb;ciaa149.

4. Adams J. Recommendation regarding the use of cloth face coverings. Especially in areas of significant community-based transmission [Internet]. [ last reviewed: April 3, 2020] Available from:

CDC for Disease Control and Prevention; 2020. Available from: https:// www.cdc.gov/coronavirus/2019-ncov/prevent-getting-sick/clothface-cover.html

5. Brienen NC, Timen A, Wallinga J, van Steenbergen JE, Teunis PF. The effect of mask use on the spread of influenza during a pandemic. Risk Anal. 2010;30(8):1210-8.

6. Aiello AE, Murray GF, Perez V, Coulborn RM, Davis BM, Uddin M, et al. Mask use, hand hygiene, and seasonal influenza-like illness among young adults: a randomized intervention trial. J Infect Dis. 2010;201(4):491-8.

7. Cooper DW, Hinds WC, Price JM, Weker R, Yee HS. Common materials for emergency respiratory protection: leakage tests with a manikin. Am Ind Hyg Assoc J. 1983;44(10):720-6.

8. van der Sande M, Teunis P, Sabel R. Professional and home-made face masks reduce exposure to respiratory infections among the general population. PLoS One. 2008;3(7):e2618. 
9. Rengasamy S, Eimer B, Shaffer RE. Simple respiratory protection — evaluation of the filtration performance of cloth masks and common fabric materials against 20-1000 nm size particles. Ann Occup Hyg. 2010;54(7):789-98.

10. Davies A, Thompson KA, Giri K, Kafatos G, Walker J, Bennett A. Testing the efficacy of homemade masks: would they protect in an influenza pandemic? Disaster Med Public Health Prep. 2013;7(4):413-8.

11. Shakya KM, Noyes A, Kallin R, Peltier RE. Evaluating the efficacy of cloth facemasks in reducing particulate matter exposure. J Expo Sci Environ Epidemiol. 2017;27(3):352-7.

12. Konda A, Prakash A, Moss GA, Schmoldt M, Grant GD, Guha S. Aerosol filtration efficiency of common fabrics used in respiratory cloth masks. ACS Nano. 2020 Apr 24:acsnano.0c03252.

13. Lane HC, Marston HD, Fauci AS. Conducting clinical trials in outbreak settings: points to consider. Clin Trials. 2016;13(1):92-5.

14. Anderson RM, Heesterbeek H, Klinkenberg D, Hollingsworth TD. How will country-based mitigation measures influence the course of the COVID-19 epidemic? Lancet. 2020;395(10228):931-4.

15. Jefferson T, Del Mar CB, Dooley L, Ferroni E, Al-Ansary LA, Bawazeer GA, van Driel ML, Nair S, Jones MA, Thorning S, Conly JM. Physical interventions to interrupt or reduce the spread of respiratory viruses. Cochrane Database Syst Rev. 2011 Jul 6;2011(7):CD006207.
16. Suess T, Remschmidt C, Schink SB, Schweiger B, Nitsche A, Schroeder $\mathrm{K}$, et al. The role of facemasks and hand hygiene in the prevention of influenza transmission in households: results from a cluster randomised trial; Berlin, Germany, 2009-2011. BMC Infect Dis. $2012 ; 12(1): 26$.

17. Liu X, Zhang S. COVID-19: face masks and human-to-human transmission. Influenza Other Respir Viruses. 2020 Mar. https://doi. org/10.1111/irv.12740.

18. Lancet T; The Lancet. COVID-19: too little, too late? Lancet. 2020;395(10226):755.

19. World Health Organization (WHO). China leaders discuss next steps in battle against coronavirus outbreak [Internet]. Genève: WHO; 2020. [updated 2020 Feb 7; cited 2020 AP 21]. Available from: https://www. who.int/news-room/detail/28-01-2020-who-china-leaders-discussnext-steps-in-battle-against-coronavirus-outbreak

20. Chinese Center for Disease Control and Prevention (CDC). Chinese center for disease control and prevention (CDC) tips of prevention for COVID-19 [Internet]. 2020 [updated 2020 Apr 21; cited 2020 Ap 21]. Available from: http://www.inacdc.cn/jkzt/crb/zl/szkb_11803/ jszl_2275/202003/t20200308_214205.html

21. Leung CC, Lam TH, Cheng KK. Mass masking in the COVID-19 epidemic: people need guidance. Lancet. 2020;395(10228):945.

\section{Anexo 1. Tabela de estudos excluídos}

1. Al-Jasser FS, Kabbash IA, AlMazroa MA, Memish ZA. Patterns of diseases and preventive measures among domestic hajjis from Central, Saudi arabia [complete republication] [complete republication]. East Mediterr Health J. 2013;19 Suppl 2:S34-41. https://doi.org/10.26719/2013.19. Supp2.S34 PMID:24673097

2. Allsopp J, Basu MK, Browne RM, Burge PS, Matthews JB. Survey of the use of personal protective equipment and prevalence of work related symptoms among dental staff. Occup Environ Med. 1997 Feb;54(2):125-34. https://doi.org/10.1136/0em.54.2.125 PMID:9072020

3. Sim SW, Moey KS, Tan NC. The use of facemasks to prevent respiratory infection: a literature review in the context of the Health Belief Model. Singapore Med J. 2014 Mar;55(3):160-7. https://doi.org/10.11622/smedj.2014037 PMID:24664384

4. Lu YT, Chen PJ, Sheu CY, Liu CL. Viral load and outcome in SARS infection: the role of personal protective equipment in the emergency department. J Emerg Med. 2006 Jan;30(1):7-15. https://doi.org/10.1016/j.jemermed.2005.03.011 PMID:16434329

5. Dato VM, Hostler D, Hahn ME. Simple respiratory mask. Emerg Infect Dis. 2006 Jun;12(6):1033-4. https://doi.org/10.3201/ eid1206.051468 PMID:16752475

6. Chughtai AA, Seale H, Dung TC, Hayen A, Rahman B, Raina Maclntyre C. Compliance with the Use of Medical and Cloth Masks Among Healthcare Workers in Vietnam. Ann Occup Hyg. 2016 Jun;60(5):619-30. https://doi.org/10.1093/annhyg/mew008 PMID:26980847

7. Tracht SM, Del Valle SY, Hyman JM. Mathematical modeling of the effectiveness of facemasks in reducing the spread of novel influenza A (H1N1). PLoS One. 2010 Feb;5(2):e9018. https://doi.org/10.1371/journal.pone.0009018 PMID:20161764

8. Rexroth U, Buda S. Praxismanagement und Arbeitsschutz während der Influenza-Pandemie 2009/2010 - Eine Umfrage unter 1150 Ärzten der Arbeitsgemeinschaft Influenza am Robert Koch-Institut. Gesundheitswesen. 2014 0ct;76(10):670-5. https://doi. org/10.1055/s-0033-1355402 PMID:24165918

9. Lau MS, Cowling BJ, Cook AR, Riley S. Inferring influenza dynamics and control in households. Proc Natl Acad Sci USA. 2015 Jul;112(29):9094-9. https://doi.org/10.1073/pnas.1423339112 PMID:26150502

10. Fahdiyani R, Raksanagara AS, Sukandar H. Influence of household environment and maternal behaviors to upper respiratory infection among toddlers. Kesmas: National Public Health Journal. 2016;10(3):120-6. https://doi.org/10.21109/kesmas.v10i3.589.

11. Lo JY, Tsang TH, Leung YH, Yeung EY, Wu T, Lim WW. Respiratory infections during SARS outbreak, Hong Kong, 2003. Emerg Infect Dis. 2005 Nov;11(11):1738-41. https://doi.org/10.3201/eid1111.050729 PMID:16318726

12. Cowling BJ, Fung RO, Cheng CK, Fang VJ, Chan KH, Seto WH, et al. Preliminary findings of a randomized trial of non-pharmaceutical interventions to prevent influenza transmission in households. PLoS One. 2008 May;3(5):e2101. https://doi.org/10.1371/journal. pone.0002101 PMID:18461182

13. Suess T, Remschmidt C, Schink SB, Schweiger B, Nitsche A, Schroeder K, et al. The role of facemasks and hand hygiene in the prevention of influenza transmission in households: results from a cluster randomised trial; Berlin, Germany, 2009-2011. BMC Infect Dis. 2012 Jan;12(1):26. https://doi.org/10.1186/1471-2334-12-26 PMID:22280120

14. Larson EL, Ferng YH, Wong-McLoughlin J, Wang S, Haber M, Morse SS. Impact of non-pharmaceutical interventions on URIs and influenza in crowded, urban households. Public Health Rep. 2010 Mar-Apr;125(2):178-91. https://doi.org/10.1177/003335491012500206 PMID:20297744

15. Cowling BJ, Chan KH, Fang VJ, Cheng CK, Fung RO, Wai W, et al. Facemasks and hand hygiene to prevent influenza transmission in households: a cluster randomized trial. Ann Intern Med. 2009 0ct;151(7):437-46. https://doi.org/10.7326/0003-4819-151-7-20091006000142 PMID:19652172
Não define o tipo de máscaras utilizadas pelos peregrinos.

Não especifica os equipamentos de proteção individual (EPI) utilizados e está direcionado para a área de odontologia.

Revisão que aborda os fatores que influenciam 0 uso de máscaras na comunidade. Não especifica o tipo de máscara.

Profissionais da saúde.

Carta ao editor ratificando o uso de máscaras

feitas em casa nas epidemias do passado.

Profissionais da saúde, máscaras cirúrgicas e de tecido.

Modelo matemático.

Profissionais de saúde.

Não utiliza máscaras de tecido feitas em casa.

Profissionais de saúde.

Intervenções em síndromes respiratórias, mas não especificamente máscaras de tecido.

Máscaras cirúrgicas.

Máscaras cirúrgicas.

Máscaras cirúrgicas.

Máscaras cirúrgicas. 
Continuação.

\begin{tabular}{|c|c|}
\hline $\begin{array}{l}\text { 16. Maclntyre CR, Zhang Y, Chughtai AA, Seale H, Zhang D, Chu Y, Zhang H, Rahman B, Wang Q. Cluster randomised controlled trial to } \\
\text { examine medical mask use as source control for people with respiratory illness. BMJ Open. } 2016 \text { Dec 30;6(12):e012330. doi: 10.1136/ } \\
\text { bmjopen-2016-012330. PMID: 28039289; PMCID: PMC5223715. }\end{array}$ & Máscaras cirúrgicas \\
\hline $\begin{array}{l}\text { 17. Macintyre CR, Seale H, Dung TC, Hien NT, Nga PT, Chughtai AA, et al. A cluster randomised trial of cloth masks compared with medical masks } \\
\text { in healthcare workers. BMJ Open. } 2015 \text { Apr;5(4):e006577-006577. https://doi.org/10.1136/bmjopen-2014-006577 PMID:25903751 }\end{array}$ & Profissionais de saúde e máscaras cirúrgicas. \\
\hline $\begin{array}{l}\text { 18. Wang M, Barasheed 0, Rashid H, Booy R, El Bashir H, Haworth E, et al. A cluster-randomised controlled trial to test the efficacy of facemasks } \\
\text { in preventing respiratory viral infection among Hajj pilgrims. J Epidemiol Glob Health. } 2015 \text { Jun;5(2):181-9. https://doi.org/10.1016/j. } \\
\text { jegh.2014.08.002 PMID:25922328 }\end{array}$ & Máscaras cirúrgicas. \\
\hline $\begin{array}{l}\text { 19. Turnberg W, Daniell W, Seixas N, Simpson T, Van Buren J, Lipkin E, et al. Appraisal of recommended respiratory infection control } \\
\text { practices in primary care and emergency department settings. Am J Infect Control. } 2008 \text { May;36(4):268-75. https://doi.org/10.1016/j. } \\
\text { ajic.2007.08.004 PMID:18455047 }\end{array}$ & Profissionais de saúde. \\
\hline $\begin{array}{l}\text { 20. Stuart RL, Gillespie EE, Kerr PG. A pilot study of an influenza vaccination or mask mandate in an Australian tertiary health service. Med J Aust. } \\
\text { 2014 Feb;200(2):83-4. https://doi.org/10.5694/mja13.10947 PMID:24484101 }\end{array}$ & $\begin{array}{l}\text { Sem desfecho de interesse, não especifica o tipo } \\
\text { de máscara. Carta ao editor. }\end{array}$ \\
\hline $\begin{array}{l}\text { 21. Wu J, Xu F, Zhou W, Feikin DR, Lin CY, He X, et al. Risk factors for SARS among persons without known contact with SARS patients, Beijing, } \\
\text { China. Emerg Infect Dis. } 2004 \text { Feb;10(2):210-6. https://doi.org/10.3201/eid1002.030730 PMID:15030685 }\end{array}$ & Não especifica o tipo de máscara. \\
\hline $\begin{array}{l}\text { 22. Zhang Y, Seale H, Yang P, Maclntyre CR, Blackwell B, Tang S, et al. Factors associated with the transmission of pandemic (H1N1) } 2009 \\
\text { among hospital healthcare workers in Beijing, China. Influenza Other Respir Viruses. } 2013 \text { May; 7(3):466-71. https://doi.org/10.1111/ } \\
\text { irv.12025 PMID:23078163 }\end{array}$ & Profissionais de saúde. \\
\hline $\begin{array}{l}\text { 23. Chughtai AA, Maclntyre CR, Ashraf MO, Zheng Y, Yang P, Wang Q, et al. Practices around the use of masks and respirators among } \\
\text { hospital health care workers in } 3 \text { diverse populations. Am J Infect Control. } 2015 \text { Oct;43(10):1116-8. https://doi.org/10.1016/j. } \\
\text { ajic.2015.05.041 PMID:26184766 }\end{array}$ & Máscaras em hospitais. \\
\hline $\begin{array}{l}\text { 24. Shimasaki N, Okaue A, Kikuno R, Shinohara K. Comparison of the Filter Efficiency of Medical Nonwoven Fabrics against Three Different } \\
\text { Microbe Aerosols. Biocontrol Sci. 2018;23(2):61-9. https://doi.org/10.4265/bio.23.61 PMID:29910210 }\end{array}$ & Estudo laboratorial com máscara cirúrgica. \\
\hline $\begin{array}{l}\text { 25. Matthews J, Slater K, Newsom SW. The effect of surgical gowns made with barrier cloth on bacterial dispersal. J Hyg (Lond). } 1985 \\
\text { Aug;95(1):123-30. https://doi.org/10.1017/S0022172400062355 PMID:4020106 }\end{array}$ & Estudo laboratorial não relacionado ao tema. \\
\hline $\begin{array}{l}\text { 26. Nagayama A. Inactivation of influenza A virus by gentian violet (GV) and GV-dyed cotton cloth, and bactericidal activities of these agents. J } \\
\text { Infect Chemother. } 2006 \text { Apr;12(2):73-9. https://doi.org/10.1007/s10156-005-0426-7 PMID:16648946 }\end{array}$ & Estudo laboratorial não relacionado ao tema. \\
\hline $\begin{array}{l}\text { 27. Maclntyre CR, Chughtai AA. Facemasks for the prevention of infection in healthcare and community settings. BMJ. } 2015 \mathrm{~A} \\
\text { doi: } 10.1136 / \mathrm{bmj} . \mathrm{h694}\end{array}$ & Não trata de máscaras de tecido feitas em casa. \\
\hline
\end{tabular}

\section{Anexo 2. Estratégias de busca}

\section{Estratégia PUBMED}

\#1 "Respiratory Tract Diseases"[Mesh] OR (Disease, Respiratory Tract) OR (Diseases, Respiratory Tract) OR (Respiratory Tract Disease) OR (Tract Disease, Respiratory) OR (Tract Diseases, Respiratory) AND

\#2 "Respiratory Protective Devices"[Mesh] OR (Device, Respiratory Protective) OR (Devices, Respiratory Protective) OR (Protective Device, Respiratory) OR (Protective Devices, Respiratory) OR (Respiratory Protective Device) OR (Respirators, Industrial) OR (Industrial Respirators) OR (Industrial Respirator) OR (Respirator, Industrial) OR (Gas Masks) OR (Gas Mask) OR (Mask, Gas) OR (Masks, Gas) OR (Respirators, Air-Purifying) OR (Air-Purifying Respirator) OR (Air-Purifying Respirators) OR (Respirator, Air-Purifying) OR (Respirators, Air Purifying) OR "Masks"[Mesh] OR (Mask*) AND

\#3 “Primary Prevention" [Mesh] OR (Disease Prevention, Primary) OR (Disease Preventions, Primary) OR (Primary Disease Prevention) OR (Primary Disease Preventions) OR (Prevention, Primary) OR (Primordial Prevention) OR (Preventions, Primordial) OR (Primordial Preventions) OR (Prevention, Primordial) OR “Secondary Prevention"[Mesh] OR (Prevention, Secondary) OR (Preventions, Secondary) OR (Secondary Preventions) OR (Secondary Disease Prevention) OR (Disease Prevention, Secondary) OR (Disease Preventions, Secondary) OR (Prevention, Secondary Disease) OR (Preventions, Secondary Disease) OR (Secondary Disease Preventions) OR (Relapse Prevention) OR (Prevention, Relapse) OR (Preventions, Relapse) OR (Relapse Preventions) OR (Early Therapy) OR (Early Therapies) or (Therapies, Early) OR (Therapy, Early)

\section{Estratégia COCHRANE LIBRARY \\ ID Search}

\#1 MeSH descriptor: [Respiratory Tract Diseases] explode all trees

\#2 (Disease, Respiratory Tract) or (Diseases, Respiratory Tract) or (Respiratory Tract Disease) or (Tract Disease, Respiratory) or (Tract Diseases, Respiratory)

\#3 \#1 or \#2

\#4 MeSH descriptor: [Respiratory Protective Devices] explode all trees

\#5 (Device, Respiratory Protective) or (Devices, Respiratory Protective) or (Protective Device, Respiratory) or (Protective Devices, Respiratory) or (Respiratory Protective Device) or (Respirators, Industrial) or (Industrial Respirators) or (Industrial Respirator) or (Respirator, Industrial) or (Gas Masks) or (Gas Mask) or (Mask, Gas) or (Masks, Gas) or (Respirators, Air-Purifying) or (Air-Purifying Respirator) or (Air-Purifying Respirators) or (Respirator, Air-Purifying) or (Respirators, Air Purifying)

\#6 \#4 or \#5

\#7 MeSH descriptor: [Masks] explode all trees

\#8 (Mask)

\#9 $\quad$ \#7 or \#8

\#10 \#6 or \#9

\#11 MeSH descriptor: [Primary Prevention] explode all trees

\#12 (Disease Prevention, Primary) or (Disease Preventions, Primary) or (Primary Disease Prevention) or (Primary Disease Preventions) or (Prevention, Primary) or (Primordial Prevention) or

(Preventions, Primordial) or (Primordial Preventions) or (Prevention, Primordial)

$\# 13 \quad \# 11$ or \#12

\#14 MeSH descriptor: [Secondary Prevention] explode all trees

\#15 (Prevention, Secondary) or (Preventions, Secondary) or (Secondary Preventions) or (Secondary Disease Prevention) or (Disease Prevention, Secondary) or (Disease Preventions, Secondary) or (Prevention, Secondary Disease) or (Preventions, Secondary Disease) or (Secondary Disease Preventions) or (Relapse Prevention) or (Prevention, Relapse) or (Preventions, Relapse) or (Relapse

Preventions) or (Early Therapy) or (Early Therapies) or (Therapies, Early) or (Therapy, Early)

\#16 \#14 or \#15

\#17 \#13 or \#16

\#18 \#3 AND \#10 AND \#17 


\section{Estratégia PORTAL REGIONAL BVS}

\#1 MH:"Doenças Respiratórias" OR (Doença \$ Respiratória\$) OR (Doença\$ do Aparelho Respiratório) OR (Doença \$ do Sistema Respiratório) OR (Doença \$ do Trato Respiratório) OR (Doença \$ das Vias Respiratórias) OR (Doença \$ do Aparelho Respiratório) OR (Doenças do Sistema Respiratório) OR (Doenças do Trato Respiratório) OR MH:C08\$ OR MH:SP4.001.012.143\$ 0R MH:SP4.046.452.698.904\$ OR (Enfermedades Respiratórias) OR (Respiratory Tract Disease\$) AND

\#2 MH:Masks OR MasK\$ OR Máscara\$ OR MH:E07.325.877.500\$ OR MH:E07.700.500\$ OR MH:E07.858.594.750\$ OR MH:J01.637.708.560.782\$ OR MH:"Respiratory Protective Devices" OR (Dispositivos de Protección Respiratoria) OR (Dispositivos de Proteção Respiratória) OR (Air-Purifying Respirator) OR (Air-Purifying Respirators) OR (Device, Respiratory Protective) OR (Devices, Respiratory Protective) OR (Gas Mask) OR (Gas Masks) OR (Industrial Respirator) OR (Industrial Respirators) OR (Mask, Gas) OR (Masks, Gas) OR (Protective Device, Respiratory) OR (Protective Devices, Respiratory) OR (Respirator, Air-Purifying) OR (Respirator, Industrial) OR (Respirators, Air Purifying) OR (Respirators, Air-Purifying) OR (Respirators, Industrial) OR (Respiratory Protective Device) OR MH:E07.700.700\$ OR MH:J01.637.708.560.937\$ OR (Máscaras de Gás) OR (Respiradores Industriais) OR (Respiradores de Ar Purificado) AND

\#3 MH:"Primary Prevention" OR (Prevención Primaria) OR (Prevenção Primária) OR (Disease Prevention Primary) OR (Disease Preventions Primary) OR (Prevention Primary) OR (Prevention Primordial) OR (Preventions Primordial) OR (Primary Disease Prevention) OR (Primary Disease Preventions) OR (Primordial Prevention) OR (Primordial Preventions) OR MH:N02.421.726.758\$ OR MH:N06.850.780.680\$ OR MH:SP2.026.182 OR MH:"Secondary Prevention" OR (Prevención Secundaria) OR (Prevenção Secundária) OR (Disease Prevention, Secondary) OR (Disease Preventions , Secondary) OR (Early Therapies) OR (Early Therapy) OR (Prevention, Relapse) OR (Prevention, Secondary) OR (Prevention, Secondary Disease) OR (Preventions, Relapse) OR (Preventions, Secondary) OR (Preventions, Secondary Disease) OR (Relapse Prevention) OR (Relapse Preventions) OR (Secondary Disease Prevention) OR (Secondary Disease Preventions) OR (Secondary Preventions) OR (Therapies, Early) OR (Therapy, Early) OR MH:E02.897\$ OR MH:N02.421.726.825\$ OR MH:N06.850.780.750\$ OR MH:SP2.026.187\$

\section{Estratégia EMBASE}

\#1 'respiratory tract disease'/exp OR (airway disease) OR (airway disorder) OR (respiration disease) OR (respiration tract disease) OR (respiratory disease) OR (respiratory disorder) OR (respiratory illness) OR (respiratory tract diseases) OR (respiratory tract disorder)

AND

\#2 'gas mask'/exp OR Gasmask OR (respiratory protective devices) OR 'mask'/exp OR mask*

\#3 'primary prevention'/exp OR 'secondary prevention'/exp

\section{Estratégia CINAHL}

\#1 (Respiratory Tract Diseases) OR (Disease, Respiratory Tract) OR (Diseases, Respiratory Tract) OR (Respiratory Tract Disease) OR (Tract Disease, Respiratory) OR (Tract Diseases, Respiratory) AND

\#2 (Respiratory Protective Devices) OR (Device, Respiratory Protective) OR (Devices, Respiratory Protective) OR (Protective Device, Respiratory) OR (Protective Devices, Respiratory) OR (Respiratory Protective Device) OR (Respirators, Industrial) OR (Industrial Respirators) OR (Industrial Respirator) or (Respirator, Industrial) OR (Gas Masks) OR (Gas Mask) OR (Mask, Gas) OR (Masks, Gas) OR (Respirators, Air-Purifying) OR (Air-Purifying Respirator) OR (Air-Purifying Respirators) OR (Respirator, Air-Purifying) OR (Respirators, Air Purifying) OR Mask ${ }^{\star}$

\section{Estratégia WEB OF SCIENCE}

\#1 (Respiratory Tract Diseases) OR (Disease, Respiratory Tract) OR (Diseases, Respiratory Tract) OR (Respiratory Tract Disease) OR (Tract Disease, Respiratory) OR (Tract Diseases, Respiratory) AND

\#2 (Respiratory Protective Devices) OR (Device, Respiratory Protective) OR (Devices, Respiratory Protective) OR (Protective Device, Respiratory) OR (Protective Devices, Respiratory) OR (Respiratory Protective Device) OR (Respirators, Industrial) OR (Industrial Respirators) OR (Industrial Respirator) or (Respirator, Industrial) OR (Gas Masks) OR (Gas Mask) OR (Mask, Gas) OR (Masks, Gas) OR (Respirators, Air-Purifying) OR (Air-Purifying Respirator) OR (Air-Purifying Respirators) OR (Respirator, Air-Purifying) OR (Respirators, Air Purifying) OR Mask ${ }^{\star}$

\#3 (Primary Prevention) OR (Disease Prevention, Primary) OR (Disease Preventions, Primary) OR (Primary Disease Prevention) OR (Primary Disease Preventions) OR (Prevention, Primary) OR (Primordial Prevention) OR (Preventions, Primordial) OR (Primordial Preventions) OR (Prevention, Primordial) OR (Secondary Prevention) OR (Prevention, Secondary) OR (Preventions, Secondary) OR (Secondary Preventions) OR (Secondary Disease Prevention) OR (Disease Prevention, Secondary) OR (Disease Preventions, Secondary) OR (Prevention, Secondary Disease) OR (Preventions, Secondary Disease) OR (Secondary Disease Preventions) OR (Relapse Prevention) OR (Prevention, Relapse) OR (Preventions, Relapse) OR (Relapse Preventions) OR (Early Therapy) OR (Early Therapies) OR (Therapies, Early) OR (Therapy, Early)

\section{Estratégia SCOPUS}

\#1 (Respiratory Tract Diseases) OR (Disease, Respiratory Tract) OR (Diseases, Respiratory Tract) OR (Respiratory Tract Disease) OR (Tract Disease, Respiratory) OR (Tract Diseases, Respiratory) AND

\#2 (Respiratory Protective Devices) OR (Device, Respiratory Protective) OR (Devices, Respiratory Protective) OR (Protective Device, Respiratory) OR (Protective Devices, Respiratory) OR (Respiratory Protective Device) OR (Respirators, Industrial) OR (Industrial Respirators) OR (Industrial Respirator) or (Respirator, Industrial) OR (Gas Masks) OR (Gas Mask) OR (Mask, Gas) OR (Masks, Gas) OR (Respirators, Air-Purifying) OR (Air-Purifying Respirator) OR (Air-Purifying Respirators) OR (Respirator, Air-Purifying) OR (Respirators, Air Purifying) OR Mask

\#3 (Primary Prevention) OR (Disease Prevention, Primary) OR (Disease Preventions, Primary) OR (Primary Disease Prevention) OR (Primary Disease Preventions) OR (Prevention, Primary) OR (Primordia Prevention) OR (Preventions, Primordial) OR (Primordial Preventions) OR (Prevention, Primordial) OR (Secondary Prevention) OR (Prevention, Secondary) OR (Preventions, Secondary) OR (Secondary Preventions) OR (Secondary Disease Prevention) OR (Disease Prevention, Secondary) OR (Disease Preventions, Secondary) OR (Prevention, Secondary Disease) OR (Preventions, Secondary Disease) OR (Secondary Disease Preventions) OR (Relapse Prevention) OR (Prevention, Relapse) OR (Preventions, Relapse) OR (Relapse Preventions) OR (Early Therapy) OR (Early Therapies) OR (Therapies, Early) OR (Therapy, Early) 